This item was submitted to Loughborough's Research Repository by the author.

Items in Figshare are protected by copyright, with all rights reserved, unless otherwise indicated.

\title{
A new family of blind adaptive equalization algorithms
}

PLEASE CITE THE PUBLISHED VERSION

PUBLISHER

(C) IEEE

VERSION

VoR (Version of Record)

LICENCE

CC BY-NC-ND 4.0

REPOSITORY RECORD

Baykal, Buyurman, Oguz Tanrıkulu, A.G. Constantinides, and Jonathon Chambers. 2019. "A New Family of Blind Adaptive Equalization Algorithms". figshare. https://hdl.handle.net/2134/5810. 
This item was submitted to Loughborough's Institutional Repository (https://dspace.lboro.ac.uk/) by the author and is made available under the following Creative Commons Licence conditions.

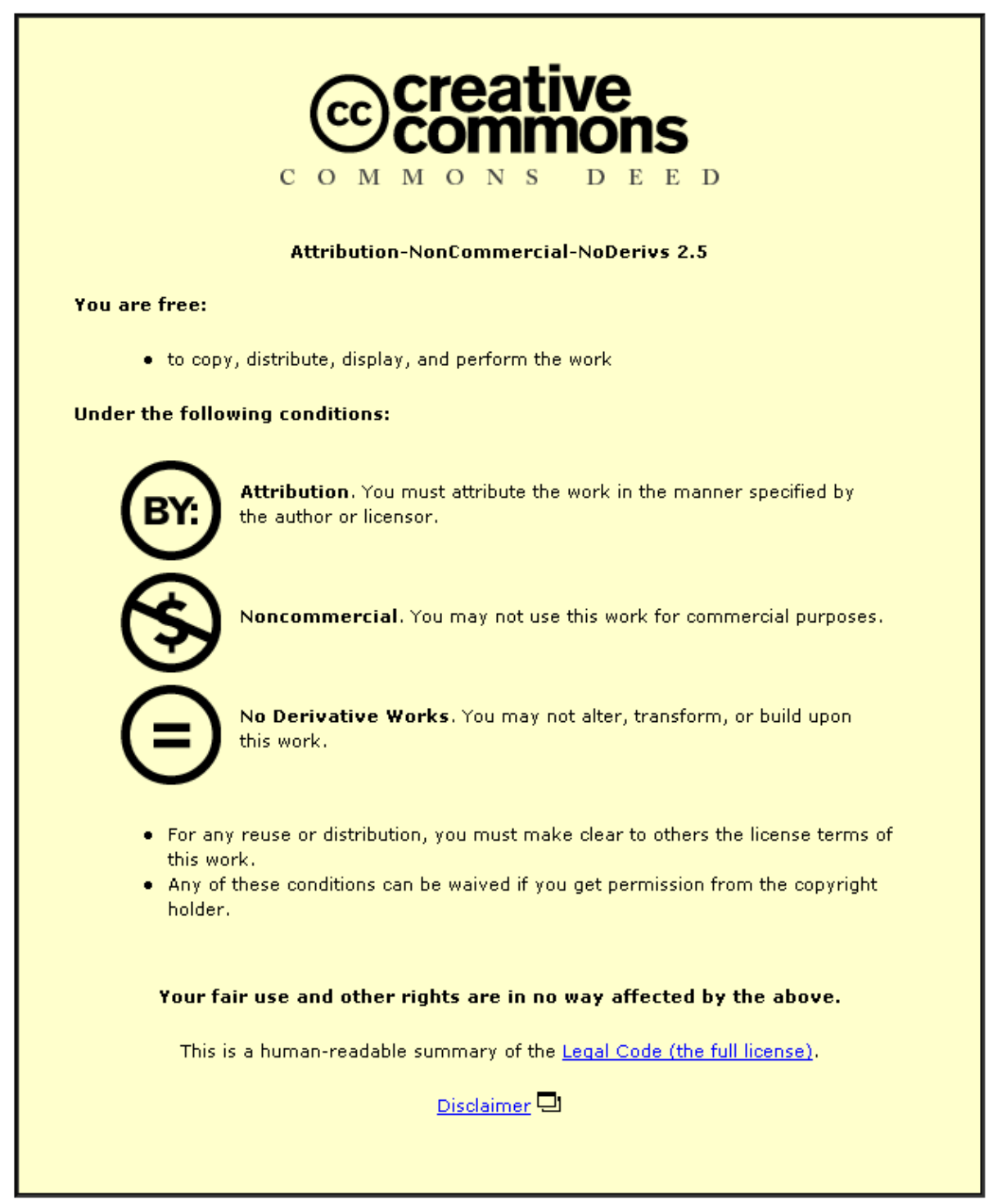

For the full text of this licence, please go to: http://creativecommons.org/licenses/by-nc-nd/2.5/ 


\title{
A New Family of Blind Adaptive Equalization Algorithms
}

\author{
Buyurman Baykal, Member, IEEE, Oğuz Tanrıkulu, Member, IEEE, Anthony G. Constantinides, Fellow, IEEE, \\ and Jonathon A. Chambers, Member, IEEE
}

\begin{abstract}
New blind adaptive channel equalization techniques based on a deterministic optimization criterion are presented. A family of nonlinear functions is proposed which constitutes a generic class of blind algorithms. They have been shown to have better performance than the conventional constant modulus algorithm (CMA)-like approaches. The advantages include the relaxed stability range on the step size and that an automatic gain control unit which estimates the gain of the channel, is no longer of crucial importance.
\end{abstract}

\section{INTRODUCTION}

$\mathbf{T}$ HE soft-constraint-satisfaction (SCS)-based algorithms have been proven to have better performance than the conventional CMA-like approaches [1]. The SCS approach in [1] includes a particular nonlinearity. In this letter, a general family of nonlinear functions have been postulated to maintain the improved performance of SCS-based approach thereby providing an infinite number of functions for use.

\section{DERIVATION OF SCS AlgORITHMS}

The rationale behind the SCS approach, contrary to the previous research of stochastic cost functions, is to use a deterministic cost function with appropriate constraints. Consider the following optimization problem, also known as the principle of minimum disturbance [2]: Determine the tap-weight vector of dimension $N$ at time $k, W_{k}$, given the tap-input vectors $X_{k}, X_{k-1}, \cdots$, and desired responses $d_{k}, d_{k-1}, \cdots$, so as to minimize the squared Euclidean norm of the change in the tap-weight vector $W_{k}$, i.e.,

$$
\min _{W_{k}}\left\|W_{k}-W_{k-1}\right\|^{2} \quad \text { subject to } X_{k}^{H} W_{k}=d_{k}
$$

where $d_{k}$ is the training sequence. In blind equalization the training sequence is not known to receiver, and hence the desired response must be obtained from pertinent quantities in the receiver. The a priori equalizer output $y_{k}=X_{k}^{H} W_{k-1}$ can be used for our purposes. Hence, the following choice for

Manuscript received June 2, 1998. The associate editor coordinating the review of this letter and approving it for publication was Prof. A. Haimovich

B. Baykal is with the Department of Electrical and Electronic Engineering, Baskent University, Baglica Kampusu, Ankara 06530, Turkey (e-mail: baykal@baskent.edu.tr).

O. Tanrikulu is with DSP Software Engineering, Inc., Bedford, MA 01730 USA.

A. G. Constantinides and J. A. Chambers are with the Department of Electrical and Electronic Engineering, Imperial College of Science, Technology and Medicine, London SW7, U.K.

Publisher Item Identifier S 1089-7798(99)04125-3.
TABLE I

The Essential Features of CM Nonlinear Functions $\psi(y): R^{1} \rightarrow R^{1}, y \in R^{1}$, cf. FIG. 1

\begin{tabular}{c|c}
\hline$\psi(y)$ is continuous and odd & $\psi(y)$ has finite derivative at the origin \\
$\psi \psi(y)>y$ for $0<y<1$ & $\psi(y)$ intersects $y$ solely at 1 and -1 \\
\hline$\psi(y)-y$ must be decreasing for $y>1$ & $\psi(y)$ must be decreasing for $0<y<1$ \\
\hline
\end{tabular}

$d_{k}$ is a viable one:

$$
d_{k}=\psi\left(y_{k}\right)
$$

where $\psi($.$) is a nonlinear function which satisfies certain$ conditions. It is possible to include multiple constraints in the derivation of algorithms, but it is beyond the scope of this letter. In [1] the particular SCS-1 nonlinearity $\psi(y)=$ $(2-|y|) y$ has been derived indirectly. Herein, a general approach is presented to include a family of functions.

The solution of the above optimization problem via Lagrange multipliers yields

$$
W_{k}=W_{k-1}+\frac{1}{X_{k}^{H} X_{k}} X_{k} e_{k}
$$

where $e_{k} \triangleq d_{k}-X_{k}^{H} W_{k-1}$. A stepsize is also introduced to maintain the stability of the algorithm. Hence, we can obtain the update of the SCS algorithms as

$$
W_{k}=W_{k-1}+\mu \frac{1}{X_{k}^{H} X_{k}} X_{k} e_{k} .
$$

The principle of minimal disturbance can be modified for use in fractionally spaced equalizers as well.

\section{A. Conditions on the Nonlinear Function $\psi(\cdot)$}

The choice of nonlinear functions $\psi(\cdot)$ is of crucial importance in the development. These functions restore the constant modulus (CM) of the communication signals. For the sake of simplicity, we assume that the communication system uses real modulation schemes. ${ }^{1}$ The essential features of CM nonlinear functions are tabulated in Table I. If the characteristics of the functions are investigated with reference to Fig. 1, it is seen that the functions should be symmetric with respect to origin (odd) because these type of equalizers cannot resolve $180^{\circ}$ of phase ambiguity. The sole intersection points $\mp 1$ correspond to the desired CM of the equalizer output. When the equalizer output is close to $\mathrm{CM}$, so is the nonlinear function and the correction term in (4) is minimal. The continuity and finite derivative properties come from the smoothness

\footnotetext{
${ }^{1}$ The functions can be used as well for complex modulation schemes but care should be taken not to neglect modulus operators.
} 


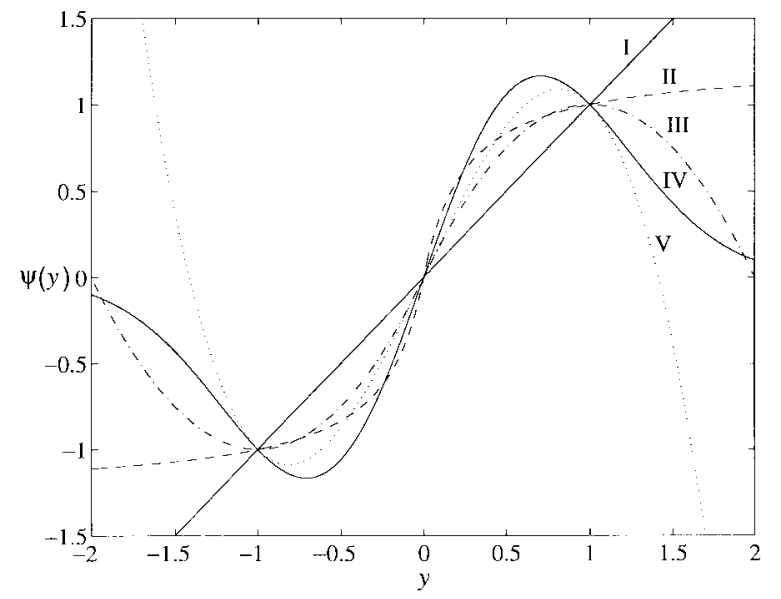

Fig. 1. Examples of $\mathrm{CM}$ functions for SCS algorithms: I) $y$ (equalizer output), II) $(y / 1-0.8(1-|y|))$, III) SCS-1: $(2-|y|) y$, IV) $y e^{-|y|^{2}+1}$, and V) Godard: $\left(2-|y|^{2}\right) y$.

and differentiability restrictions. The other properties about the range of $y$ define the requirements for the algorithm to converge to the CM. For instance, $\psi(y)>y$ for $0<y<1$ guarantees that the correction term in (4) pushes the algorithm toward $\mathrm{CM}$ when $0<y<1$.

An infinite number of functions can be found to satisfy the properties in Table I, which provide flexibility in the design of blind equalizers. For instance, in limited precision arithmetic processors higher powers of the input signal can be avoided. Convergence speed and estimation error are dependent on the choice of the nonlinear function. At low signal-to-noise ratio (SNR) values, the shape of the nonlinear function is less critical.

\section{SiMULATIONS}

We simulated a $T$-spaced equalizer with three taps in a fading scenario with three algorithms which result from the nonlinearities III-V in Fig. 1. Also, we have included the unnormalized versions, i.e., the normalization in (4) have been removed. The open-eye-measure $(\mathrm{OEM})^{2}$ [1] for each

${ }^{2}$ The OEM for channel equalizers is defined as $\operatorname{OEM}(k) \triangleq\left(\left\|T_{k}\right\|_{1}\right.$ $\left.\left\|T_{k}\right\|_{\infty} /\left\|T_{k}\right\|_{\infty}\right)$ where $T_{k}$ represents the combined channel and equalizer space. If $\operatorname{OEM}(k)<0 \mathrm{~dB}$ then the eye is open and ISI has no effect in the decision process. If $\operatorname{OEM}(k)>0 \mathrm{~dB}$, the eye is closed and hence the ISI left after equalization will cause decision errors. The lower the OEM, the better approximation of the inverse channel the equalizer provides.
TABLE II

Step Sized (Top Row) and Mean OEM's (dB) (Bottom Row) in Each Slot. QAM Approximation of GMSK, Three-Ray Channel Model with Average Powers of 0.1, 0.8, ANd 0.3 AT INTEger Delays, SNR of $40 \mathrm{~dB}$, Bit Rate of $271 \mathrm{~kb} / \mathrm{s}, 950 \mathrm{MHz}$ CARrier FreQuency AND Mobile SPEed of $150 \mathrm{~km} / \mathrm{h}$

\begin{tabular}{|c|c|c|c|c|c|c|c|}
\hline \multirow[b]{2}{*}{ Unnormalized IV } & 0.01 & 0.02 & 0.03 & 0.04 & 0.05 & 0.06 & 0.07 \\
\hline & -4.618 & -5.374 & -5.108 & -7.050 & -7.179 & -4.609 & -5.372 \\
\hline \multirow[b]{2}{*}{ IV (c.f. Figure 1) } & 0.07 & 0.08 & 0.09 & 0.1 & 0.125 & 0.15 & 0.175 \\
\hline & -6.776 & -6.929 & -7.026 & -7.088 & -7.166 & -6.923 & -6.914 \\
\hline \multirow[b]{2}{*}{ Unnormalized V (Godard Algorithm [2]) } & 0.009 & 0.01 & 0.02 & 0.03 & 0.04 & 0.05 & 0.06 \\
\hline & -4.689 & -5.806 & -6.636 & -6.698 & -6.956 & $\mathrm{NaN}$ & $\mathrm{NaN}$ \\
\hline \multirow[b]{2}{*}{$V$ (c.f. Figure 1) } & 0.07 & 0.08 & 0.09 & 0.1 & 0.125 & 0.15 & 0.175 \\
\hline & -3.442 & -7.846 & -7.930 & -7.911 & -7.841 & -7.826 & $\mathrm{NaN}$ \\
\hline \multirow[b]{2}{*}{ Unnormalized III } & 0.01 & 0.02 & 0.03 & 0.04 & 0.05 & 0.06 & 0.07 \\
\hline & -5.987 & -4.657 & -6.813 & -5.695 & -5.761 & -7.159 & -6.913 \\
\hline \multirow[b]{2}{*}{ III (SCS-1 Algorith } & 0.125 & 0.15 & 0.175 & 0.2 & 0.225 & 0.25 & 0.275 \\
\hline & -7.5 .56 & -7.697 & -7.771 & -7.826 & -7.641 & -7.729 & 7.745 \\
\hline
\end{tabular}

algorithm is averaged over 30000 samples in $\mathrm{dB}$ scale. The runs are identical for each of the algorithms. The initial convergence periods have been excluded in the computations. The results in Table II demonstrate the improved tracking capability of the SCS algorithms in fading environments. Time-varying channel taps cause singularities frequently in the channel, i.e., zeros of the channel cross the unit circle, which means that it is impossible to equalize the channel. Equalizer taps fluctuate during singularities. Good tracking algorithms recover quickly and reconverge to optimum values. In that sense the SCS algorithms have a faster response to changes in the channel. The lower the OEM, the better the algorithms track the time-varying channel on average. Also, the SCS algorithms maintain tracking capability over a wider range of stepsizes, whereas the unnormalized counterparts have their best performance in a limited range.

\section{CONCLUSIONS}

A family of nonlinear functions is proposed as an extension of the SCS approach [1] which form a new set of blind adaptive algorithms. They are observed to maintain the advantages of the SCS approach.

\section{REFERENCES}

[1] O. Tanrikulu, B. Baykal, A. Constantinides, and J. Chambers, "Soft constraint satisfaction (SCS) blind channel equalization algorithm," Int. J. Adaptive Contr. Signal Processing, vol. 12, no. 2, pp. 117-134, 1998.

[2] B. Widrow and M. Lehr, "30 years of adaptive neural networks: Perceptron, madaline and backpropagation," Proc. IEEE, vol. 78, pp. 1415-1442, 1990. 